\title{
Estrategias cognitivas ejecutadas en la resolución de problemas matemáticos en una prueba de admisión a la educación superior
}

\section{Cognitive strategies performed in the resolution of mathematical problems in a test of admission to higher education}

\author{
Randall Blanco-Benamburg, ${ }_{1}^{1}$ Katherine Palma-Picado, ${ }^{2}$ \\ Tania Elena Moreira-Mora ${ }^{3}$
}

\begin{abstract}
Resumen: El propósito del estudio fue analizar las estrategias cognitivas ejecutadas en la resolución de ítems matemáticos de la prueba de aptitud académica (PAA) del Instituto Tecnológico de Costa Rica (ITCR), desde un enfoque metodológico descriptivo y transversal. La principal técnica para la recolección de datos fue la entrevista cognitiva, con el uso de un protocolo validado con un panel de expertos y un grupo focal. Entre los principales resultados se encontró que los participantes no ejecutan la totalidad de las tareas en cada una de las etapas de resolución: comprender el problema, concebir un plan, ejecutar el plan y verificar. También se encontraron diferencias en la ejecución de las tareas entre los tipos de razonamiento: inductivo, deductivo, resolución de problemas, probabilístico y con figuras. Estos hallazgos pueden ser una respuesta a la problemática del bajo desempeño del estudiantado en pruebas enfocadas en estrategias heurísticas al brindar información específica sobre la ruta de resolución, utilizada por estudiantes de alto desempeño matemático, a partir de un conjunto de tareas cognitivas que se pueden desarrollar en el aula.
\end{abstract}

Fecha de recepción: 18 de octubre de 2019. Fecha de aceptación: 09 de septiembre de 2020.

${ }^{1}$ Instituto Tecnológico de Costa Rica, rblanco@itcr.ac.cr, orcid.org/0000-0002-0984-1768

2 Instituto Tecnológico de Costa Rica, kpalma@itcr.ac.cr, orcid.org/0000-0003-3323-5714

3 Instituto Tecnológico de Costa Rica, tmoreira@itcr.ac.cr, orcid.org/0000-0002-8955-0804 
Palabras clave: estrategias cognitivas, pruebas estandarizadas, razonamiento matemático, entrevista cognitiva, educación superior.

\begin{abstract}
The purpose of the study was to analyze the cognitive strategies executed in the resolution of the mathematic items for the test of academic abilities from the Instituto Tecnológico de Costa Rica (ITCR), from a methodologic descriptive and cross approach. The principal technique for collecting the data was with a cognitive interview, with the use of a protocol validated for a panel of experts and a focal group. Between the principal results, was found that the participants did not execute the total tasks on each one of the steps of resolution: understand the problem, conceive and execute a plan and verify it. In addition, differences were found in the execution of tasks between the types of reasoning: inductive, deductive, problem resolution, probabilistic and with figures. From a pedagogical standpoint, this research could point to a solution for the low performance rates in tests that include heuristics in that it provides specific information related to problem solving routes used by high achieving students. This understanding could guide classroom instruction.
\end{abstract}

Keywords: cognitive strategies, standardized testing, math reasoning, cognitive interview, higher education.

\title{
1. INTRODUCCIÓN
}

El Instituto Tecnológico de Costa Rica (ITCR) es una institución de educación superior pública, que ha aplicado la prueba de aptitud académica (PAA) desde su apertura en 1973 para seleccionar a los candidatos. Esta prueba está conformada por dos componentes: matemático y verbal, cuyos ítems se han enfocado en la medición de las habilidades de razonamiento en ambas áreas.

En el 2009 se realizó una investigación descriptiva con la finalidad de identificar esas habilidades verbales y matemáticas y ajustar la tabla de especificaciones de la PAA al perfil de entrada de los estudiantes y a las nuevas demandas cognoscitivas de las carreras para garantizar una muestra representativa de estas habilidades (Moreira-Mora, 2010). No obstante, el proceso de validación involucra la acumulación de evidencia relevante para proveer una amplia base científica para la interpretación de las puntuaciones de acuerdo 
con los propósitos de su uso. De ahí surgió la necesidad de obtener nuevas evidencias relacionadas con las estrategias de razonamiento usadas por los examinados en la resolución de problemas matemáticos.

Román y Carbonero (2002, citados por Carbonero y Coromoto, 2006), plantean que la deficiente utilización de estrategias cognitivas y metacognitivas es una de las causas de los fracasos de los alumnos y por lo tanto se requiere contemplar en la instrucción matemática, el desarrollo de heurísticos o estrategias para analizar o resolver conflictos, razonamiento inductivo e intuitivo, y la comprobación de hipótesis (p. 349). La identificación de estas estrategias cognitivas permite obtener evidencias asociadas a los procesos de respuesta.

Según Padilla y Benitez (2014), la mayoría de los estudios de validación se han enfocado en las evidencias asociadas al contenido y al constructo; no obstante, los estudios basados en los procesos de respuesta de los participantes fueron estudiados solo en 1,8\% de los artículos analizados. Según estos autores, la mayoría de estos estudios han sido en el área de salud y, en general, buscan identificar los elementos de los ítems que pueden causar desajustes entre los procesos de respuesta y las especificaciones delineadas en la prueba.

A pesar de la relevancia de las estrategias cognitivas en la resolución de pruebas estandarizadas de ingreso a las universidades costarricenses como la Universidad de Costa Rica (UCR), Universidad Nacional (UNA) y el ITCR, en el país no se han llevado a cabo estudios que permitan identificar las estrategias ejecutadas por los estudiantes al resolver estos ítems. Únicamente se ha realizado un estudio relacionado con las habilidades verbales de la prueba de aptitud académica de la UCR y la UNA (Brizuela, Jiménez, Pérez y Rojas, 2016); por lo tanto, el objetivo del presente estudio fue analizar las estrategias cognitivas ejecutadas por los estudiantes para la resolución de ítems de matemática de la PAA del ITCR.

\section{ANTECEDENTES}

A partir del estudio de Valle, Juárez y Guzmán (2007) con estudiantes de la Olimpiada Mexicana de Matemática, se identificaron dificultades para comprender los problemas planteados, así como un mejor desempeño en la definición de estrategias de solución entre quienes habían recibido entrenamiento previo. Para el análisis de los datos recurrieron a descripción verbal de las estrategias, 
al cálculo de la frecuencia de uso de ellas y de la incidencia en las distintas áreas de la matemática a la que pertenecían los problemas.

Vergel, Duarte y Martínez (2015) desarrollaron una investigación que consistió en correlacionar la planificación de estrategias para la enseñanza de las matemáticas con el desarrollo del pensamiento matemático de los estudiantes de cálculo integral de universidades de Santander; concluyeron que las actividades del docente para mejorar la comprensión de los temas de mayor dificultad, está relacionada con el nivel de desarrollo de pensamiento. Al respecto Marugán, Martín, Catalina y Román (2012), señalan que la elaboración de una estrategia cognitiva facilita una mejor comprensión, retención y recuperación informativa, de tal forma que a mayor elaboración mayor codificación y recuperación de la información.

En otro estudio con estudiantes mexicanos que inician carreras universitarias y otros que están concluyendo su formación se encontró que el desempeño cognitivo mejora cuando: el estudiante se enfrenta a problemas que le son familiares, cuando hay conexión entre la capacidad del estudiante para representar gráficamente una situación mediante dibujos, diagramas, gráficas o esquemas lo que a su vez constituye un mecanismo de autocontrol del propio razonamiento (Waldegg y Agüero, 1999).

\section{MARCO TEÓRICO}

\subsection{ESTRATEGIAS DE RESOLUCIÓN DE PROBLEMAS}

En general, las estrategias cognitivas ayudan al individuo a enfocar su atención en las cuestiones relevantes o significativas de la tarea que se le presenta; lo motivan a establecer conexiones, a procesar y a reorganizar la información de manera profunda (Woolfolk, 2010). Es así como la mente del ser humano trabaja formando representaciones mentales a las cuales se les aplica procesos cognitivos que aseguran la obtención del conocimiento favoreciendo la codificación y almacenamiento de información, su recuperación posterior y su utilización en la solución de problemas (Osses y Jaramillo, 2008).

En el campo particular de la matemática, las estrategias cognitivas se han definido como los procesos que el individuo elige frente a una tarea matemática con el propósito de facilitar la adquisición, el almacenamiento y/o la utilización de información o conocimientos (Sanjurjo y Vera, 1994). Para Valle, 
Cabanach, Rodríguez, Nuñez y González-Pienda (2006) existen diferentes clasificaciones para designar las estrategias cognitivas, aunque para el caso de resolución de problemas matemáticos hay coincidencia en que las básicas son:

- De organización: implica la lectura comprensiva de enunciados para identificar el problema, organizar los datos, establecer prioridades y buscar relaciones.

- De formulación: consiste en la exploración que hace el individuo para considerar diferentes opciones de solución, definir un plan a seguir y ejecutarlo.

- De selección: referido a la elección que hace el individuo sobre cuál tipo de razonamiento aplicar. De acuerdo con Beltrán (2002), la selección implica diferenciar entre y dentro de las fuentes de información según la importancia y relevancia de criterio.

- De memorización: implica el proceso de recuperación de información (Woolfolk, 2010).

Sobre el estudio de estrategias para resolver problemas matemáticos son reconocidos los trabajos de Polya (1987), quien considera que la experiencia previa de la persona y la observación de la forma como otros resuelven problemas son insumos importantes. Además, afirma que las heurísticas utilizadas en el proceso de resolución no dependen del contenido involucrado en el problema que se pretenda resolver. Su modelo, propuesto en 1965, considera cuatro pasos para la solución de problemas:

1. Comprender el problema: Se debe comprender el enunciado, separar las partes principales, la incógnita, los datos, la condición, hacer una figura si corresponde, introducir la notación adecuada y preguntarse si el problema tendrá solución.

2. Concebir un plan: En esta etapa se deben identificar cálculos, razonamientos o construcciones que deberán realizarse para determinar la solución. En este proceso es útil preguntarse: si se conoce un problema relacionado, si puede enunciarse de modo diferente, si conviene primero resolver un problema similar más simple.

3. Ejecución del plan: Para ejecutar el plan diseñado se requiere, además de concebir la idea, utilizar conocimientos previos, concentración, hábitos de pensa- 
miento y paciencia. La implementación puede conducir a la solución o a una nueva estrategia.

4. Visión retrospectiva: Una vez ejecutado el plan y redactada la solución es recomendable que se revise el trabajo realizado, para confirmar la respuesta correcta a lo que se cuestionó, incluso si se puede resolver el problema de otra manera.

\subsection{HABILIDADES DE RAZONAMIENTO MATEMÁTICO}

Los ítems de razonamiento matemático de la PAA del ITCR se clasifican en las siguientes categorías:

- Resolución de problemas: El examinado debe hacer uso de conocimientos básicos, definir y ejecutar una estrategia de solución. La solución de un problema, como señalan Marino y Rodríguez (2015) "implica un proceso creativo y de una complejidad cognitiva mayor, en tanto que el alumno debe elaborar su propio método de resolución, apelando a sus conocimientos previos, estableciendo nuevas relaciones entre ellos $y$, además, empleando diversos procedimientos, tanto algorítmicos como heurísticos" (p. 161).

- Deductiva: Según Lassiter y Goodman (2015), el razonamiento deductivo "implica procedimientos matemáticamente bien definidos para extraer las consecuencias que siguen con certeza o necesidad de algún tipo de evidencia" (p. 124). Mediante la deducción se conduce de forma sistemática de un grupo de proposiciones a otra.

- Inductiva: Mediante la inducción se pueden crear nuevos conceptos analizando semejanzas o diferencias, este razonamiento "se hace visible a través de operaciones como clasificar, completar series, hacer analogías y comparaciones con diferentes tipos de símbolos (verbales, figuras, entre otros), que permiten llegar a hacer inferencias para definir esos nuevos conceptos y posteriormente aplicarlos y evaluarlos" (Iriarte, Espeleta, Zapata, Cortina, Zambrano y Fernández, 2010, p. 42). Este razonamiento se caracteriza por ampliar la información de la que se parte y por la comprobación de la validez de la nueva información (Cañadas y Castro, 2006). 
- Probabilística: implica el análisis de situaciones que no han ocurrido y tienen más de un resultado probable. La capacidad de pensar a partir de las probabilidades es considerada un tipo de razonamiento particular, el cual es muy útil en la toma de decisiones en la vida cotidiana (Nickerson, 2004, citado por Erdem y Gürbüz, 2016, p. 39).

- Con figuras: está relacionada con la habilidad de percibir formas y transformarlas mentalmente. Según Smith (2009), citada por Noriega, Vásquez y García (2011, p. 98), la visualización espacial se caracteriza por "la capacidad de ver, concebir, manipular mentalmente los objetos del mundo visual y realizar transformaciones a partir de lo percibido; distinguiéndola de la mera habilidad o memoria visual, que es una forma estática o reproductiva de visualización".

\section{MÉTODO}

En función del objetivo de esta investigación se optó por un diseño descriptivo y transversal, la técnica de la entrevista cognitiva y un protocolo para la recolección de los reportes verbales y las respuestas sobre las tareas cognitivas.

La técnica se basa en entrevistas uno a uno realizadas con apoyo de un cuestionario guía (Jobe, 2003); que permite al entrevistado revelar los motivos de sus respuestas, la elaboración de ellas, las dificultades en la comprensión de las preguntas y las tareas de recuperación de información, entre otras (Briceño, Álvarez, Barco, Álvarez, Delgado y Zúñiga, 2016, p. 191). Al respecto Smith y Molina (2011) señalan que al entrevistado se le piden dos cosas básicas: decir en voz alta todo lo que está pensando mientras completa el cuestionario (autorreporte verbal) y contestar una serie de preguntas (pruebas cognitivas de reporte verbal). Como resalta Hernández (2002), el pensamiento en voz alta permite al investigador hacer patentes comportamientos representativos de los procesos complejos que se dan en la solución de problemas y, además, ofrece una idea de la estructura y la forma en que trabaja la mente.

En cuanto a la construcción del protocolo fue necesario la revisión de literatura y un riguroso proceso de validación como se describe en las siguientes etapas del procedimiento metodológico. 


\subsection{Primera fase: Definición DE LAS CATEgorías de LAS habilidadeS MATEMÁTICAS MEDIDAS EN LA PAA}

Para definir las categorías se efectuaron las siguientes actividades:

a) Revisión de la literatura para la descripción conceptual de las categorías de razonamiento matemático.

b) Especificación de cada categoría con sus correspondientes etapas y tareas cognitivas.

\subsection{SEgunda FASE: CONSTRUCCIÓN Y VALIDACIÓN DEL PROTOCOLO}

En la primera etapa se trabajó con un primer panel de jueces con el propósito de revisar la estructura y contenidos de los componentes del protocolo, mientras que en la segunda etapa se realizó una aplicación piloto del instrumento para ser analizada por un segundo panel de expertos.

El juicio de expertos se define como una opinión informada de personas con trayectoria en el tema, que son reconocidas por otros como expertos cualificados en este y que, pueden dar información, evidencia, juicios y valoraciones (Escobar-Perez y Cuervo-Martinez, 2008).

La primera etapa fue realizada en marzo del 2018 con la colaboración de 9 jueces con la finalidad de revisar la estructura y contenidos de los componentes del protocolo. Entre este equipo de jueces se contó con dos en el área de la psicología cognitiva y otros siete con amplia experiencia en la construcción de pruebas estandarizadas en matemática.

Para esta etapa se construyó una guía con las siguientes secciones: introducción, datos generales del experto, instrucciones, definiciones de las categorías de razonamiento matemático y una tabla para calificar la coherencia de las tareas cognitivas basada en el modelo de Cuatro Etapas propuesto por Tourangeau, así como de la estructura del protocolo.

A manera de resumen, las observaciones hechas por los 9 jueces, tanto al instrumento del protocolo como al consentimiento informado de los participantes, se centraron en aspectos de contenido y formato. Específicamente, las principales recomendaciones al protocolo fueron: 
- Realizar con los jueces participantes de la fase siguiente el análisis de un ítem de la aplicación piloto para que ellos comprendan el constructo de razonamiento matemático y sus categorías.

- Revisar las definiciones de las categorías e incorporar ejemplos de ítems matemáticos.

- Permutar algunas tareas entre las etapas, incluir otras nuevas y algunas relacionadas con lo heurístico.

- Brindar una definición de cada etapa del modelo para la construcción del protocolo.

- Entre las recomendaciones para mejorar el desempeño de los estudiantes durante la entrevista cognitiva se destacan las siguientes:

- Incluir un ejercicio matemático para practicar el reporte verbal, antes de la aplicación de la entrevista cognitiva.

- Tratar de simplificar el nivel de complejidad de las tareas para facilitar su comprensión y reducir el listado en algunas etapas.

- Facilitar una ficha con el listado de las tareas para que los estudiantes puedan seguir la lectura del investigador.

- Utilizar un vocabulario menos técnico para facilitar la comprensión de los ítems.

Finalmente, se revisaron algunos aspectos de formato y de redacción en todas las secciones del protocolo. Tales recomendaciones fueron consideradas tanto para la aplicación piloto de la segunda etapa como para mejorar la estructura y contenidos del protocolo.

La segunda etapa de la validación inició con la aplicación piloto del instrumento para evaluar la relevancia, la suficiencia, el contenido y la representatividad de las preguntas y las tareas cognitivas descritas en el protocolo, en cada una de las categorías del razonamiento matemático. Esta aplicación fue realizada en abril del 2018 con cuatro estudiantes que ingresaron al ITCR ese año. Esta muestra estaba constituida por 3 hombres y una mujer, provenientes de dos colegios públicos, uno subvencionado y otro privado.

Para esta etapa se contó con la colaboración de 8 jueces, quienes cumplían con el perfil requerido y participaron en dos talleres. El primer taller fue en mayo 2018 para explicar la metodología de trabajo, la técnica de la entrevista cognitiva con el uso del protocolo y las categorías de razonamiento matemático de la PAA. El segundo, se realizó en junio de ese mismo año para el análisis de las descripciones conceptuales de las categorías de razonamiento matemático y sus tareas cognitivas. En 
esta sesión los jueces compartieron sus observaciones individuales de los 8 ítems seleccionados del folleto de práctica de la PAA del 2017 que representaban todas las categorías. Entre las recomendaciones derivadas de esta discusión grupal se destacan:

- Revisar en las tareas cognitivas: el orden, la pertinencia con la categoría o la etapa, la claridad y la incorporación de otras tareas específicas a cada tipo de razonamiento.

- Revisar en las preguntas abiertas: la redacción, agregar y replantear algunas para indagar más en los procesos mentales del participante.

- Separar el razonamiento deductivo y el inductivo para diferenciarlas por sus tareas cognitivas específicas.

- Del modelo de Cuatro Etapas de Tourangeau utilizado en el protocolo, valorar la opción de eliminar la etapa de estimación porque se confundía con recuperación de la información.

- Ampliar las tareas de la etapa de comprensión y de verificación.

- Señalar en la transcripción los tiempos de las pausas durante la resolución del ítem.

Tales recomendaciones fueron incluidas para mejorar el protocolo y la técnica de la entrevista cognitiva antes de la aplicación principal. Además de estas mejoras, el cambio más relevante fue la transición del modelo de Tourangeau hacia el de cuatro pasos de Polya (1987), ya que, de esta forma se evitaba la confusión entre la etapa de recuperación de la información y la estimación, cuyas tareas están contenidas en el paso de comprender el problema de Polya, quien además considera la verificación de la respuesta; etapa relevante en cualquier estrategia de resolución de problemas matemáticos.

Una vez concluida esta segunda fase, el protocolo quedó organizado en tres partes. En la primera se describe brevemente la técnica de la entrevista cognitiva y los objetivos. Luego, en la parte de entrenamiento, el participante realiza un ejercicio matemático de baja dificultad para ensayar el reporte verbal. La tercera incluía el listado de las preguntas abiertas (pruebas cognitivas de reporte verbal) y las tareas cognitivas por cada categoría de razonamiento matemático. En esta sección los participantes tenían la oportunidad de reflexionar sobre lo ejecutado en cada una de las etapas, además del autoreporte verbal inicial. Como lo indica Hernández (2002, p. 42), se exploró no sólo lo que reside en las mentes de los participantes, sino que se estudiaron sus estrategias en la solución de problemas y su reflexión sobre las mismas. 


\subsection{TERCERA FASE: APLICACIÓN PRINCIPAL DE LA ENTREVISTA COGNITIVA}

El marco de la muestra estaba constituido por 174 estudiantes que obtuvieron una nota igual o mayor a 90 en el componente de matemática de la PAA del 2017, que se caracterizaba por estar conformada por 79,19\% de hombres y $20,81 \%$ de mujeres, quienes provenían el 41,52\% de colegios privados, el 36,84\% de públicos y el $21,63 \%$ de subvencionados. De esta lista de estudiantes se seleccionaron 18 de manera aleatoria, quienes habían matriculado cursos en el segundo semestre del 2018.

Los criterios técnicos para la selección de los ítems fueron los siguientes: tener un nivel de dificultad intermedio, entre $40 \%$ y $60 \%$ de acierto, mostrar una discriminación igual o mayor a 0,30 y representar diversas tareas de las estrategias de razonamiento de cada categoría, como se resume en la tabla 1.

Tabla 1. Descripción de los ítems utilizados en la aplicación principal de la entrevista cognitiva.

\begin{tabular}{|c|c|c|c|c|}
\hline Ítems seleccionados & $\begin{array}{l}\text { Índice de } \\
\text { dificultad }\end{array}$ & $\begin{array}{c}\text { Índice de } \\
\text { discriminación }\end{array}$ & $\begin{array}{l}\text { Total de } \\
\text { reportes } \\
\text { verbales }\end{array}$ & $\begin{array}{l}\text { Distribución de } \\
\text { los jueces }\end{array}$ \\
\hline Inductivo $N^{\circ} 1$ & 49 & 0,46 & 10 & $\mathrm{~J} 3$ y J4 \\
\hline Inductivo $\mathrm{N}^{\circ} 2$ & 45 & 0,33 & 8 & $\mathrm{~J} 3$ y J4 \\
\hline Deductivo $N^{\circ} 3$ & 62 & 0,49 & 8 & $\mathrm{~J} 1$ y J2 \\
\hline Deductivo $N^{\circ} 4$ & 47 & 0,36 & 10 & $\mathrm{~J} 2$ y J3 \\
\hline Resolución de problemas $N^{\circ} 5$ & 58 & 0,49 & 6 & $\mathrm{~J} 5$ y J6 \\
\hline Resolución de problemas $N^{\circ} 6$ & 46 & 0,44 & 6 & $\mathrm{~J} 5$ y J6 \\
\hline Resolución de problemas $N^{\circ} 7$ & 52 & 0,48 & 6 & $\mathrm{~J} 5$ y J6 \\
\hline Con figuras $N^{\circ} 8$ & 54 & 0,34 & 6 & 17 y 18 \\
\hline Con figuras $N^{\circ} 9$ & 57 & 0,35 & 6 & $\mathrm{J7}$ y $\mathrm{J} 8$ \\
\hline Con figuras $\mathrm{N}^{\circ} 10$ & 47 & 0,27 & 6 & $\mathrm{~J} 7$ y 18 \\
\hline Probabilístico Nº 11 & 42 & 0,41 & 6 & J9 y J4 \\
\hline Probabilístico Nº 12 & 37 & 0,32 & 6 & J2 y J9 \\
\hline Probabilístico N 13 & 42 & 0,52 & 6 & J9 y J1 \\
\hline
\end{tabular}


Como se observa, el ítem $\mathrm{N}^{\circ} 1$ resultó fácil y el $\mathrm{N}^{\circ} 12$ difícil; mientras que el $N^{\circ} 10$ mostró un índice de discriminación menor a 0,30; aun así, se consideraron aceptables para la aplicación principal de la investigación.

El objetivo de esta evaluación fue juzgar de manera integral la relevancia, la suficiencia y la representatividad de las tareas cognitivas ejecutadas por los participantes en cada etapa, según la categoría de razonamiento matemático. En este juzgamiento se eliminó el dominio de contenido por recomendación de los jueces en el proceso de validación del protocolo. En esta evaluación colaboró un panel de 9 expertos en la enseñanza de la matemática en educación superior, de los cuales 6 habían participado en la fase piloto. A cada uno de los jueces se le asignó tres ítems con sus correspondientes reportes verbales. La estrategia de trabajo se desarrolló de la siguiente manera:

a) Revisión de las transcripciones por parte de los investigadores.

b) Asignación de cada reporte verbal a dos jueces.

c) Organización de los reportes verbales por ítem en un archivo de Word.

d) Elaboración de la guía de juzgamiento de las respuestas ejecutadas por los participantes (solución, preguntas y tareas) con base en los siguientes dominios:

- La suficiencia del conjunto de tareas en cada etapa (comprensión, elaboración, ejecución y verificación).

- La relevancia de cada tarea en cada etapa.

- La representatividad del conjunto de tareas de cada etapa con la categoría de razonamiento (inductivo, deductivo, resolución de problemas, figuras y probabilístico).

e) Preparación de las carpetas digitales de trabajo por categoría y por juez, con los archivos en Word que contenían los reportes verbales de los ítems y una hoja de cálculo preparada para registrar las evaluaciones; más una carpeta física con las hojas de los ítems resueltos (material confidencial de la PAA) y la guía de juzgamiento de las tareas ejecutadas por los participantes.

f) Inducción general a los expertos el 12 de noviembre del 2018 para explicar el procedimiento de análisis y la guía de juzgamiento.

g) Juzgamiento individual de las estrategias de razonamiento ejecutadas por los participantes en cuatro sesiones presenciales realizadas entre el 12 y el 26 de noviembre con los expertos. 
Para determinar el grado de acuerdo entre los jueces en la evaluación de cada una de las tareas cognitivas según etapa y categoría de razonamiento matemático, se utilizó la medida de acuerdo Kappa, calculada con el paquete Statistical Package for the Social Sciences (SPSS) 19, cuyos resultados se resumen en la tabla 2. Para la interpretación de este coeficiente se utilizaron los parámetros recomendados en la literatura (Landis y Koch, 1977, p. 165): pobre (menor a 0$)$, ligero $(0-0.20)$, justo $(0.21-0.40)$, moderado $(0.41-0.60)$, sustancial $(0.61$ - 0.80) y casi perfecto $(0.81-1)$.

Tabla 2. Coeficiente Kappa de Cohen del juzgamiento de las tareas ejecutadas por etapa y categoría de razonamiento.

\begin{tabular}{lcc}
\hline Aplicación principal & $\begin{array}{c}\text { Medida de acuerdo } \\
\text { Kappa }\end{array}$ & $\begin{array}{c}\text { Nivel de } \\
\text { significancia }\end{array}$ \\
\hline Inductivo $\mathrm{N}^{\circ} 1$ & .417 & .000 \\
Inductivo $\mathrm{N}^{\circ} 2$ & .361 & .004 \\
Deductivo $\mathrm{N}^{\circ} 3$ & .258 & .030 \\
Deductivo $\mathrm{N}^{\circ} 4$ & .506 & .000 \\
Resolución de problemas $N^{\circ} 5$ & .289 & .013 \\
Resolución de problemas $N^{\circ} 6$ & .193 & .093 \\
Resolución de problemas $\mathrm{N}^{\circ} 7$ & .308 & .005 \\
Con figuras $\mathrm{N}^{\circ} 8$ & .452 & .000 \\
Con figuras $\mathrm{N}^{\circ} 9$ & .482 & .000 \\
Con figuras $\mathrm{N}^{\circ} 10$ & .541 & .000 \\
Probabilístico $\mathrm{N}^{\circ} 11$ & .417 & .001 \\
Probabilístico $\mathrm{N}^{\circ} 12$ & .146 & .176 \\
Probabilístico $\mathrm{N}^{\circ} 13$ & .501 & .000 \\
\hline
\end{tabular}

En general, los coeficientes Kappa oscilaron entre los valores justos $(0,21-0,40)$ y moderados $(0,41$ - 0,60). De acuerdo con Escobar-Pérez y Cuervo-Martínez (2008, p. 32), "Io común es obtener un amplio espectro de valores intermedios que se interpretan teniendo como referencia la complejidad de la evaluación y el número de categorías a evaluar". Según estos resultados se rechaza la $\mathrm{H}_{0}$ cuando el valor observado excede al valor crítico (con un $\alpha$ de 0.05 ); por lo 
tanto, se puede concluir que hay acuerdo entre los expertos, el valor de k muestra la proporción de acuerdo eliminando el acuerdo que podría ser efecto del azar, con excepción de dos ítems: resolución de problemas $\mathrm{N}^{\circ} 6$ y razonamiento probabilístico $\mathrm{N}^{\circ} 12$.

\section{RESULTADOS}

La estrategia de análisis de esta investigación se fundamentó en la triangulación de la información obtenida en diferentes fuentes: Ios reportes verbales de la entrevista cognitiva, las soluciones escritas de los estudiantes en los folletos de examen, las observaciones del equipo de investigadores y las evaluaciones de los jueces. Como lo destacan Okuda y Gómez-Restrepo (2005), la triangulación ofrece la alternativa de poder visualizar un problema desde diferentes ángulos, le confiere a un estudio rigor, profundidad, complejidad y permite dar grados variables de consistencia a los hallazgos.

En función del objetivo de esta investigación se describen de manera individual cada una de las categorías de razonamiento matemático, según las etapas del modelo teórico de Polya, para destacar las particularidades y similitudes en las estrategias ejecutadas por los participantes en los diferentes ítems.

En la categoría de razonamiento deductivo se incluyeron dos ítems, uno basado en un silogismo y el otro en un algoritmo, de los que debían deducir una conclusión que con certeza fuera verdadera. Estos ítems se aplicaron a un total de 12 estudiantes, quienes durante el reporte verbal y las preguntas abiertas (pruebas cognitivas) expresaron sus estrategias de razonamiento. 
Tabla 3. Valores absolutos y relativos de las tareas cognitivas ejecutadas en razonamiento deductivo.

\begin{tabular}{lcc}
\hline Tareas & Total & Porcentaje \\
\hline Comprensión & 12 & 100 \\
T1: Identificó información clave. & 10 & 83 \\
T2: Relacionó la pregunta con ejercicios previos. & 11 & 92 \\
T3: Recordó conceptos matemáticos o fórmulas. & & \\
Concebir el plan & 10 & 83 \\
T1: Relacionó información clave con algún procedimiento cono- & & \\
cido. & 11 & 17 \\
T2: Definió una estrategia de solución. & 2 & 100 \\
T3: Representó información de otra manera. & & 100 \\
Ejecutar el plan & 12 & 100 \\
T1: Ejecutó la estrategia de solución inicial. & 12 & 92 \\
T2: Relacionó premisas utilizando conectores lógicos. & 12 & 58 \\
T3: Dedujo nuevas proposiciones verdaderas. & 11 & 75 \\
Verificación & & 83 \\
T1: Comprobó la estrategia seleccionada. & 7 & \\
T2: Comprobó cada paso ejecutado. & 9 & \\
T3: Volvió a leer el enunciado. & 10 & \\
T4: Lo resolvió de otra manera. & & \\
T5: Descartó opciones. & 11 \\
\hline
\end{tabular}

Como se muestra en la tabla 3, las tres tareas de la etapa de comprensión fueron ejecutadas por la mayoría de los participantes. Por ejemplo, al consultarles si relacionó la pregunta con ejercicios resueltos, uno manifestó:

E9: De hecho, en Español con las tablas de verdad.

En general, se observó que algunos participantes necesitaron volver a leer el ítem para comprenderlo. 
En la etapa siguiente relacionada con la elaboración del plan, solo ejecutaron dos tareas; en tanto que la representación de la información de manera diferente fue ejecutada solo por dos participantes. Una posible explicación es que el ítem de silogismo es difícil representarlo de otra manera y el segundo estaba organizado en forma gráfica. Además, como lo indica uno de los jueces:

J1: "Es después de leer la pregunta que se le ocurre utilizar las respuestas para descartar y llegar a la respuesta. El ensayo y error fue parte de lo que definió su estrategia".

En general, la elaboración del plan se centra en relacionar la información clave con algún procedimiento conocido y definir una estrategia rápida, que no necesariamente aparece de forma inmediata.

En la ejecución del plan, las tres tareas fueron ejecutadas por todos, lo que demuestra que son relevantes para la deducción de nuevas proposiciones verdaderas y el uso de conectores lógicos.

Finalmente, en la verificación resalta que solo un estudiante utilizó otra estrategia para comprobar la respuesta; a pesar de ser una tarea muy pertinente para confirmar que la respuesta sea la correcta. En tanto que las tareas más frecuentes fueron volver a leer el enunciado y descartar opciones, como bien lo expresa uno de los participantes:

\section{E17: Descartar opciones lo que hace es hacer más rápido.}

En suma, los participantes no realizan todas las tareas de verificación, probablemente, por el poco tiempo disponible para resolverlo. Además, el hecho de que los ítems son de selección única y al encontrar entre las opciones la respuesta obtenida asumen que está correcta; por lo que no verifican el procedimiento.

Desde el punto de vista de los jueces, la tarea 4 de la etapa de verificación no fue relevante en el trabajo realizado por los participantes y con un nivel bajo de relevancia las tareas 3 de concebir el plan, 1 y 2 de verificación.

En la categoría de razonamiento inductivo se incluyeron dos ítems, en los cuales debían identificar un patrón para generar nuevos términos de una secuencia. Las tareas ejecutadas por los 12 participantes se resumen en la tabla 4. 
Tabla 4. Valores absolutos y relativos de las tareas cognitivas ejecutadas en razonamiento inductivo.

\begin{tabular}{|c|c|c|}
\hline Tareas & Total & Porcentaje \\
\hline \multicolumn{3}{|l|}{ Comprensión } \\
\hline T1: Identificó información clave. & 12 & 100 \\
\hline T2: Relacionó la pregunta con ejercicios previos. & 12 & 100 \\
\hline T3: Recordó conceptos matemáticos o fórmulas. & 12 & 100 \\
\hline T4: Identificó patrones. & 12 & 100 \\
\hline \multicolumn{3}{|l|}{ Concebir el plan } \\
\hline T1: Relacionó la información clave con algún procedimiento conocido. & 9 & 75 \\
\hline T2: Definió una estrategia de solución. & 12 & 100 \\
\hline T3: Representó la información de otra manera. & 4 & 33 \\
\hline \multicolumn{3}{|l|}{ Ejecutar el plan } \\
\hline T1: Ejecutó la estrategia de solución inicial. & 9 & 75 \\
\hline T2: Analizó semejanzas o diferencias para identificar un patrón. & 12 & 100 \\
\hline T3: Obtuvo una fórmula para el término general de la sucesión. & 3 & 25 \\
\hline T4: Calculó términos desconocidos de una sucesión. & 6 & 50 \\
\hline \multicolumn{3}{|l|}{ Verificación } \\
\hline T1: Comprobó la estrategia seleccionada. & 7 & 58 \\
\hline T2: Comprobó cada paso ejecutado. & 8 & 67 \\
\hline T3: Volvió a leer el enunciado. & 8 & 67 \\
\hline T4: Lo resolvió de otra manera. & 3 & 25 \\
\hline T5: Descartó opciones. & 6 & 50 \\
\hline
\end{tabular}

Tal como se observa en la tabla 4, todos ejecutaron las 4 tareas de comprensión. En la etapa de concebir el plan, el relacionar información clave con algún procedimiento conocido y definir una estrategia de solución fueron las tareas más frecuentes y la menos frecuente fue representar la información de otra manera porque no era necesario. En ambos se requería identificar un patrón, en uno de ellos se debía encontrar un término desconocido y trabajar con él para luego responder, mientras que en el otro, lo que debían seleccionar era un término de la sucesión. 
En la etapa de ejecutar el plan dos tareas son las más frecuentes, en especial, la tarea 2 que fue utilizada por todos. Ninguno de los ítems seleccionados para esta categoría requería encontrar una fórmula general.

En la última etapa, las tres primeras tareas fueron las más utilizadas, al igual que en la categoría de razonamiento deductivo. En uno de los ítems descartar opciones fue una tarea ejecutada por todos, mientras que en el otro no era necesario. Por ejemplo un participante indica:

E14: ...Lo que se puede hacer es verificar si en todos los datos de la sucesión coincide el mismo patrón ...

Los jueces no consideraron relevante la tarea 3 de la etapa de ejecución y clasificaron en un nivel bajo las tareas 3 de concebir el plan y 4 y 5 de verificación.

En la categoría de resolución de problemas se trabajó con tres ítems en los cuales era necesario realizar algunas operaciones aritméticas y cuya solución podría encontrarse por diferentes caminos. Por ejemplo, podía ser útil el uso de ecuaciones de primer grado con una incógnita, el apoyo con diagramas o el análisis de casos particulares para deducir la solución general. Estos ítems fueron realizados por un total de 18 participantes, cuyas tareas ejecutadas se resumen en la tabla 5.

Tabla 5. Valores absolutos y relativos de las tareas cognitivas ejecutadas en resolución de problemas.

\begin{tabular}{lcc}
\hline Tareas & Total & Porcentaje \\
\hline Comprensión & 18 & 100 \\
T1: Identificó información clave. & 13 & 72 \\
T2: Relacionó la pregunta con ejercicios previos. & 12 & 67 \\
T3: Recordó conceptos matemáticos o fórmulas. & 7 & 39 \\
T4: Formuló el problema de otra manera. & & \\
Concebir el plan & 16 & 89 \\
T1: Definió una estrategia de solución. & 13 & 72 \\
T2: Planteó una operación o ecuación. & 11 & 61 \\
T3: Representó la información de otra manera. & & \\
\hline
\end{tabular}




\begin{tabular}{lcc}
\hline Tareas & Total & Porcentaje \\
\hline Ejecutar el plan & 13 & 72 \\
T1: Ejecutó la estrategia de solución inicial. & 17 & 94 \\
T2: Realizó operaciones aritméticas o ecuaciones. & 8 & 44 \\
T3: Simplificó una expresión. & 9 & 50 \\
T4: Hizo una lista de datos. & 10 & 56 \\
T5: Ordenó la información. & 7 & 39 \\
T6: Utilizó una representación gráfica. & & \\
Verificación & 15 & 83 \\
T1: Comprobó la estrategia seleccionada. & 16 & 89 \\
T2: Comprobó cada paso ejecutado. & 12 & 67 \\
T3: Volvió a leer el enunciado. & 8 & 44 \\
T4: Lo resolvió de otra manera. & 6 & 33 \\
T5: Descartó opciones. & & \\
\hline
\end{tabular}

Como se puede observar, todos ejecutaron la tarea 1 de comprensión. Las tareas 2, 3 y 4 fueron realizadas por la mayoría, pero solamente 7 optaron por un planteamiento alternativo del problema. El 72\% de ellos consideró que anteriormente había realizado algún ítem similar, por ejemplo:

E14: Para unas más complejas ya tenía un procedimiento que había aprendido, es uno muy sencillo, usted empieza a dibujar cajitas, entonces al final usted suma resta los residuos y lo divide entre las cajitas, eso ayuda mucho.

Las tres tareas de la etapa concebir un plan fueron realizadas por la mayoría. A diferencia de los ítems de razonamiento deductivo e inductivo, en la resolución de problemas sí recurrieron a otras formas de representar la información. Por ejemplo, el uso del lenguaje algebraico, como lo comenta uno de ellos:

E16: Yo lo leí y ya sabía que $\mathrm{R}$ iba a ser una suma de varios números, entonces si yo ponía todos esos números en relación al primero podía obtenerlo a partir de una fórmula. 
En la etapa de ejecución la tarea más realizada fue resolver operaciones aritméticas o ecuaciones. En tanto que, no más de la mitad de ellos realizó alguna representación gráfica o simplificó una expresión. Entre 56\% y 92\% realizó las otras tres tareas. Uno de los participantes muestra cómo recurrió a trabajo aritmético y algebraico:

E5: Lo que hice fue empezar con el dato que me daban y fui restando, sumando y dividiendo o multiplicando. Me dio una respuesta que no estaba entre las opciones, entonces me fui por otro método que es plantearlo con una variable.

En la etapa de verificación menos de la mitad optó por descartar opciones para poder encontrar la respuesta correcta y solo la mitad intentó resolver el problema de una segunda forma para verificar la respuesta obtenida. Al menos dos terceras partes realizaron las primeras tres tareas. Un participante describe de la siguiente manera su trabajo de verificación:

E4: Uno podría plantearse una ecuación, pero creo que hubiera sido bastante más complicado haberla planteado así. Creo que la mejor forma de comprobarlo es con un ejemplo.

Sobre las tareas de resolución de problemas, los jueces consideraron relevantes todas las tareas, pero con un nivel bajo la 3 de concebir el plan, la 6 de ejecución y la 4 y 5 de verificación.

La característica particular de la categoría de razonamiento con figuras es la habilidad de percibir formas y transformarlas mentalmente. En el primero de los ítems utilizados los participantes debían identificar el porcentaje sombreado de una figura, en el segundo reconocer un patrón a partir de una secuencia de figuras para generar un nuevo elemento y en el tercero comparar el área de dos figuras descritas en el enunciado. En la tabla 6 se muestran los datos de las tareas ejecutadas en esta categoría de razonamiento. 
Tabla 6. Valores absolutos y relativos de las tareas cognitivas ejecutadas en razonamiento con figuras

\begin{tabular}{|c|c|c|}
\hline Tareas & Total & Porcentaje \\
\hline \multicolumn{3}{|l|}{ Comprensión } \\
\hline T1: Identificó información clave. & 18 & 100 \\
\hline T2: Recordó conceptos matemáticos o fórmulas. & 18 & 100 \\
\hline T3: Recordó procedimientos aplicados en ejercicios similares. & 12 & 67 \\
\hline T4: Representó la información de otra manera. & 12 & 67 \\
\hline T5: Realizó anotaciones o resaltó elementos de la figura. & 13 & 72 \\
\hline T6: Integró partes de una figura para construirla. & 8 & 44 \\
\hline T7: Buscó semejanzas y diferencias entre figuras de una secuencia. & 7 & 39 \\
\hline T8: Identificó diferentes posiciones o simetrías. & 9 & 50 \\
\hline \multicolumn{3}{|l|}{ Concebir el plan } \\
\hline T1: Definió una estrategia de solución. & 18 & 100 \\
\hline T2: Determinó si necesitaba calcular: área, perímetro, volumen o longitud. & 6 & 33 \\
\hline T3: Seleccionó una fórmula. & 6 & 33 \\
\hline T4: Dividió en regiones según alguna propiedad. & 5 & 28 \\
\hline T5: Agrupó regiones de una figura. & 6 & 33 \\
\hline T6: Determinó secciones de una figura tridimensional. & 0 & 0 \\
\hline T7: Dio un orden particular. & 11 & 61 \\
\hline T8: Pensó en otra variación de la figura. & 11 & 61 \\
\hline \multicolumn{3}{|l|}{ Ejecutar el plan } \\
\hline T1: Ejecutó la estrategia de solución inicial. & 16 & 89 \\
\hline T2: Visualizó la figura en otra posición, dimensión o proporción. & 8 & 44 \\
\hline T3: Aplicó una fórmula. & 6 & 33 \\
\hline T4: Agregó elementos nuevos. & 5 & 28 \\
\hline T5: Aplicó propiedades de simetrías. & 6 & 33 \\
\hline \multicolumn{3}{|l|}{ Verificación } \\
\hline T1: Comprobó la estrategia seleccionada. & 14 & 78 \\
\hline T2: Comprobó cada paso ejecutado. & 14 & 78 \\
\hline T3: Volvió a leer el enunciado. & 14 & 78 \\
\hline T4: Lo resolvió de otra manera. & 5 & 28 \\
\hline T5: Descartó opciones. & 2 & 11 \\
\hline
\end{tabular}


En la etapa de comprensión las tareas más relevantes fueron las dos primeras, enfocadas en la identificación de información y conceptos claves. Algunas no eran necesarias para esta muestra de items, tales como las tareas 6, 7 y 8; aunque sí para otros tipos de ítems de este razonamiento.

En la siguiente etapa, la tarea que fue ejecutada por los 18 participantes fue la elaboración de una estrategia, las tareas 4 y 5 resultaron irrelevantes, pues no fueron ejecutadas por la mayoría de los participantes en alguno de los tres ítems, a diferencia de la 2 y 3 que sí fueron ejecutadas por quienes resolvieron el tercer ítem. En lo que respecta a la tarea 6 no se puede afirmar que sea irrelevante, puesto que ningún ítem contenía una figura tridimensional. En este sentido uno de los jueces manifiesta:

J8: El estudiante no confeccionó ninguna figura de referencia, entonces muchas de las tareas dadas no las realizó, pero a mi criterio esto no significa que las tareas no sean relevantes.

De las 5 tareas de ejecución del plan, la más importante fue la realización de la estrategia definida inicialmente $y$, a pesar de que algunas tareas presentan baja frecuencia, sí son relevantes para la resolución de los ítems. La aplicación de propiedades de simetrías resultó irrelevante para la muestra de ítems.

En la etapa final de verificación, las 3 primeras tareas fueron las más utilizadas para la comprobación de la estrategia, los pasos y revisión del enunciado, como lo expresa un participante:

E4. Asegurándome de que los datos que puse inicialmente fueran los correctos, que me daban en el ejercicio. iEh!, comprobé la multiplicación, era una multiplicación bastante sencilla...

Desde el punto de vista de los jueces, fueron relevantes las dos primeras tareas de la etapa de comprensión, la primera de concebir el plan y de ejecución y las tres primeras de verificación.

En los ítems de razonamiento probabilístico resultaba importante que los estudiantes identificaran el carácter aleatorio de las situaciones planteadas. En dos de los ítems era necesario calcular probabilidades mediante la definición clásica y en el otro debían identificar los casos probables y determinar cuáles eventos tenían mayor probabilidad de ocurrir. 
Tabla 7. Valores absolutos y relativos de las tareas cognitivas ejecutadas en razonamiento probabilístico

\begin{tabular}{lll}
\hline Tareas & Total & Porcentaje \\
\hline
\end{tabular}

\section{Comprensión}

T1: Identificó información clave. 17 94

T2: Recordó conceptos matemáticos o fórmulas. 18

T3: Recordó procedimientos aplicados en ejercicios similares.

T4: Representó la información de otra manera.

$9 \quad 50$

T5: Reconoció casos posibles y favorables.

\section{Concebir el plan}

T1: Definió una estrategia de solución.

T2: Elaboró un diagrama.

T3: Resumió información mediante una lista o tabla.

528

T4: Planteó una estrategia donde aplique: técnicas de conteo, definición o propiedades de probabilidad, operaciones con conjuntos.

\section{Ejecutar el plan}

T1: Ejecutó la estrategia de solución inicial.

T2: Dedujo información de un diagrama.

T3: Aplicó reglas de conteo.

T4: Calculó probabilidades.

$5 \quad 28$

T5: Utilizó propiedades de probabilidad.

$15 \quad 83$

T6: Aplicó operaciones con conjuntos.

$\begin{array}{ll}7 & 39\end{array}$

\section{Verificación}

T1: Comprobó la estrategia seleccionada. $\quad 10 \quad 56$

T2: Comprobó cada paso ejecutado. $\quad 11 \quad 61$

T3: Volvió a leer el enunciado. $\quad 13 \quad 72$

T4: Lo resolvió de otra manera. $\quad 0 \quad 0$

T5: Descartó opciones. $\quad 3 \quad 17$ 
Como se muestra en la tabla 7, todas las tareas de la etapa de comprensión fueron ejecutadas por al menos la mitad de los participantes. En uno de los ítems representar la información de una manera alternativa no resultó importante, probablemente porque ya se les presentaba en una tabla. Una dificultad presentada estuvo relacionada con la comprensión de que el evento era aleatorio y no podía seguir una secuencia. Esto lo resalta uno de los jueces:

J9: El estudiante había escogido la respuesta correcta desde el inicio. Sin embargo, luego, comienza a analizar los datos obtenidos como un patrón.

En la etapa de concebir el plan, los entrevistados definieron una estrategia tomando en cuenta conceptos de probabilidad, conteo o conjuntos. Las tareas relacionadas con representación de información mediante diagramas, tablas o listas no fueron utilizadas por la mayoría. Es importante señalar que dos fallaron un mismo ítem para el que no ejecutaron la mayoría de las tareas, como indica un juez:

J2: Los estudiantes que fallaron en plantear la estrategia fallaron en la respuesta correcta.

En la etapa de ejecutar el plan solamente las tareas 1 y 4 fueron realizadas por la mayoría. Las tareas 2 y 5 resultaron relevantes solo para uno de los ítems.

En la etapa de verificación llama la atención que ningún participante intentó una segunda estrategia de solución para comprobar la respuesta. Solo 3 de ellos descartaron opciones. Las restantes tareas sí fueron realizadas por la mayoría, como lo expresa uno de ellos:

E3: Devolviéndome a leer el problema para ver que todos los datos los había apuntado bien, que había hecho la suma del total, que había visto cuántos eran los hombres de pelo negro, para tener los casos favorables y los casos posibles bien y ver que había hecho la división bien.

En la etapa de comprensión los jueces consideraron relevantes todas, excepto la 4. En las etapas de concebir el plan y de ejecutarlo indicaron que son relevantes las tareas 1 y 4, en la fase de verificación consideraron relevantes la 2 y la 3. 


\section{CONCLUSIONES}

En relación con la etapa de la descripción conceptual se encontró una diversidad de definiciones en la literatura, de manera que, se asumieron aquellas más apropiadas a la medición de las habilidades de razonamiento inductivo, deductivo, resolución de problemas, probabilístico y de figuras en una prueba estandarizada de aptitud académica. Para algunos autores estas categorías están incluidas en un concepto más amplio como el de resolución de problemas. No obstante, esta clasificación permitió identificar diferencias en la ejecución de las tareas según las categorías de razonamiento.

En cuanto a los hallazgos de la entrevista cognitiva se destaca que es la estrategia más apropiada para obtener evidencias de los procesos de respuesta y comprender la ejecución de las tareas desde el punto de vista de los entrevistados. Como lo destacan Briceño et al. (2016) esta técnica permite determinar los procesos cognitivos encubiertos y manifiestos que el entrevistado utiliza para responder. Para lograr este propósito fue necesario garantizar la calidad del protocolo de la entrevista, ya que, esto permitió evaluar la relevancia, la suficiencia y la representatividad de las preguntas y las tareas cognitivas descritas en cada una de las categorías del razonamiento matemático de una manera objetiva y más precisa. También fue acertado el criterio de selección de los participantes, lo que favoreció su desempeño en la entrevista.

De los principales resultados se tiene que la tarea más relevante ejecutada en la etapa de comprensión fue la identificación de la información clave que se utilizó en todas las categorías de razonamiento. También recordar conceptos matemáticos, fórmulas y procedimientos fue relevante para los ítems: inductivo, probabilístico, con figuras y resolución de problemas. Al respecto, Hernández (2002, p. 143) señala que en la primera lectura la mayoría de los estudiantes identifican lo que se pregunta en el problema, luego los datos que les provee para entenderla y tratan de relacionarla con algo visto anteriormente o con el conocimiento que tienen. Por otra parte, hay tareas muy específicas como fue el caso de identificar patrones para inductivo.

En la etapa de concebir el plan, señalar una estrategia para resolver el problema fue utilizada en todas las categorías. En el caso de tareas específicas relevantes, se encontró en razonamiento inductivo y deductivo relacionar la información con un procedimiento conocido; en probabilístico usar técnicas de conteo, definición y propiedades de la probabilidad y operaciones con conjuntos; en resolución de problemas plantear una operación o una ecuación y en 
razonamiento con figuras ordenar y variar la figura. Al respecto, Iriarte et al. (2010) indican que el procedimiento para la resolución de un problema empieza con tratar de comprender qué debe hacerse, segundo entender la información que se tiene para buscar la solución y tercero buscar estrategias adecuadas para llegar con esta información a la solución.

En la etapa de ejecución se encontró que la estrategia de solución inicial se implementó en todas las categorías de razonamiento. Además, de las siguientes tareas específicas: realizar operaciones aritméticas o una ecuación en resolución de problemas; visualizar la figura de otra manera; calcular probabilidades, identificar patrones y relacionar premisas.

En todas las categorías de razonamiento se incluyeron las mismas tareas de verificación, de las cuales las más relevantes fueron comprobar la estrategia seleccionada, los pasos ejecutados y releer el enunciado. Solamente en razonamiento deductivo 92\% de los participantes descartaron opciones para encontrar la respuesta. En esta etapa la evaluación tiene dos propósitos: una justificar los resultados conseguidos; otra confirmar los objetivos alcanzados (Iriarte, 2002).

En definitiva, estos resultados pueden contribuir a la labor docente para desarrollar habilidades de razonamiento matemático, ya que brinda información específica sobre la ruta de resolución utilizada por estudiantes de alto desempeño, de manera que se trascienda el enfoque mecánico en la enseñanza de la matemática, como lo afirman Pérez y Ramírez (2008).

\section{REFERENCIAS}

Beltrán, J. (2002). Procesos, estrategias y técnicas de aprendizaje. Ed. Síntesis.

Briceño, A., Álvarez, C., Barco, B., Álvarez, K., Delgado, I. y Zúñiga, V. (2016). Entrevistas cognitivas y su utilidad en la adaptación y validación de escalas para niños y adolescentes. Revista Electrónica Científica y Académica de Clínica Alemana, 6, 190-195. Brizuela, A., Jiménez, K., Pérez, N. y Rojas, G. (2016). Autorreportes verbales en voz alta para la identificación de procesos de razonamiento en pruebas estandarizadas. Revista Costarricense de Psicología, 35(1), 17-30.

Cañadas, M. C. y Castro, E. (2006). Un procedimiento para la caracterización de estrategias en problemas de sucesiones que involucran el razonamiento inductivo. Indivisa, IV, 13-24.

Carbonero, M. A. y Coromoto, J. (2006). Entrenamiento de alumnos de Educación Superior en estrategias de aprendizaje en matemáticas. Psicothema, 18(3), 348-352. 
Erdem, E. y Gürbüz, R. (2016). Evaluation of Probabilistic Reasoning Evidence from Seventh-graders. Educational Research Quarterly, 40(2), 36-66.

Escobar-Pérez, J. y Cuervo-Martínez, A. (2008). Validez de contenido y juicio de expertos: una aproximación a su utilización. Avances en Medición, 6, 27-36.

Hernández, A. (2002). Procesos cognoscitivos y metacognoscitivos en estudiantes universitarios puertorriqueños en la solución de problemas matemáticos no típicos. (Tesis doctoral no publicada). Universidad de Puerto Rico.

IBM Corporation (2016). IBM SPSS Statistics for Windows, Version 19.0. IBM Corporation. Iriarte, F., Espeleta, A., Zapata, E., Cortina, L., Zambrano, E. y Fernández, F. (2010). El razonamiento lógico en estudiantes universitarios. Zona próxima, 12.

Jobe, J. B. (2003). Cognitive psychology and self-reports: Models and methods. Quality of Life Research, 12, 219-227.

Juan-Espinosa, M. (1997). Geografía de la inteligencia humana. Las aptitudes cognitivas. Ediciones Pirámide.

Landis, J. R., y Koch, G. G. (1977). The measurement of observer agreement for categorical data. Biometrics, 33(1), 159-174.

Lassiter, D. y Goodman, N. (2015). How many kinds of reasoning? Inference, probability and natural language semantics. Cognition, 136, 123-134. https://doi.org/10.1016/j. cognition.2014.10.016

Marino, T. y Rodríguez, M. (2009). Un estudio exploratorio sobre heurísticas en estudiantes de un curso de matemática de nivel pre-universitario. Paradigma, XXX(2), 159-178.

Marugán, M., Martín L. J., Catalina, J., Román, J. M. (2012). Estrategias cognitivas de elaboración y naturaleza de los contenidos universitarios. Psicología Educativa Revista de los Psicológos de la Educación, 19(1), 13-20. https://doi.org/10.5093/ed2013a3

Moreira-Mora, T. E. (julio, 2010). Tabla de especificaciones: Una experiencia de validación de la prueba de aptitud académica del Tecnológico de Costa Rica. En Federación Iberoamericana de Asociaciones de Psicología, VII Congreso Iberoamericano de Psicología, Oviedo, España.

Noriega, M., Vásquez, S. y García, S. (2011). Componentes de la competencia espacial. Exploración en ingresantes a la facultad de Arquitectura, diseño y urbanismo. Revista de Orientación Educacional, 25(47), 95-112.

Osses, S. y Jaramillo, S. (2008). Metacognición: un camino para aprender a aprender. Estudios Pedagógicos, XXXIV(1), 187-197.

Okuda, M. y Gómez-Restrepo, C. (2005). Métodos en investigación cualitativa: triangulación. Revista Colombiana de Psiquiatría, 24(1), 118-124.

Padilla, J. L. y Benítez, I. (2014) Validity evidence based on response processes. Psicothema, 26(1), 136-144. http//doi.org/10.7334/psicothema2013.259 
Pérez, Y. y Ramírez, R. (2008). Desarrollo instruccional sobre estrategias de enseñanza de la resolución de problemas matemáticos dirigido a docentes de primer grado de Educación Básica. Caso Colegio San Ignacio. (Tesis de post-grado no publicada), Universidad Pedagógica.

Polya, G. (1987). Cómo plantear y resolver problemas. Editorial Trillas.

Sanjurjo L. y Vera M. (1994). Aprendizaje significativo y enseñanza en los niveles medio y superior. Editorial Homo Sapiens.

Smith, V. y Molina, M. (2011). Cuaderno metodológico 5. La entrevista cognitiva: Guía para su aplicación en la evaluación y mejoramiento de instrumentos de papel y lápiz.

Tourangeau, R. (1984). Cognitive science and survey methods. In Jabine, T.; Straf, M.; Tanur, J., Cognitive Aspects of survey methodology: Building a Bridge between Disciplines. National Academy Press.

Valle, A., Cabanach, R. G., Rodríguez, S., Núñez, J. C. y González-Pienda, J. A. (2006). Metas académicas, estrategias cognitivas y estrategias de autorregulación del estudio. Psicothema, 18(2), 165-170.

Valle, M. C., Juárez, M. A. y Guzmán, M. E. (2007). Estrategias generales en la resolución de problemas de la olimpiada mexicana de matemáticas. Revista Electrónica de Investigación Educativa, 9(2), 2-11.

Vergel, M., Duarte, H., y Martínez, J. (2015). Desarrollo del pensamiento matemático en estudiantes de cálculo integral su relación con la planificación docente. Revista Científica, 23, 17-29. https://doi.org/10.14483/udistrital.jour.RC.2015.23.a2

Waldegg, G. y Agüero, M. (1999). Habilidades cognoscitivas y esquemas de razonamiento en estudiantes universitarios. Revista Mexicana de Investigación Educativa, 4(8), 203-244.

Woolfolk, A. (2010). Psicología Educativa (11a. edición) (L. Pineda Trad.). Prentice Hall.

\section{RANDALL BLANCO-BENAMBURG}

Dirección: Costa Rica, Cartago, Instituto Tecnológico de Costa Rica, Escuela de Matemática, apartado postal: 159-7050

Teléfono: (506) 25502693 